ISSN 2079-9292

www.mdpi.com/journal/electronics

Review

\title{
A Brief Review of Heavy-Ion Radiation Degradation and Failure of Silicon UMOS Power Transistors
}

\author{
Kenneth F. Galloway \\ Vanderbilt University, Department of Electrical Engineering and Computer Science and \\ Institute for Space and Defense Electronics, Nashville, TN 37235-1824, USA; \\ E-Mail: Ken.Galloway@Vanderbilt.edu; Tel.: +1-615-343-0312 \\ External Editor: Mostafa Bassiouni
}

Received: 5 July 2014; in revised form: 15 August 2014 / Accepted: 16 September 2014 /

Published: 30 September 2014

\begin{abstract}
Silicon VDMOS power MOSFET technology is being supplanted by UMOS (or trench) power MOSFET technology. Designers of spaceborne power electronics systems incorporating this newer power MOSFET technology need to be aware of several unique threats that this technology may encounter in space. Space radiation threats to UMOS power devices include vulnerabilities to SEB, SEGR, and microdose. There have been relatively few studies presented or published on the effects of radiation on this device technology. The S-O-A knowledge of UMOS power device degradation and failure under heavy-ion exposure is reviewed.
\end{abstract}

Keywords: power transistors; VDMOS; UMOS; MOSFET; radiation effects; space electronics; microdose; SEB; SEGR

\section{Introduction}

The natural space radiation environment is complex, containing high-energy photons, protons, neutrons, electrons, and heavy ions. Energetic heavy ions introduce transient, dense charge filaments along their path through an electronic device that can trigger catastrophic failure mechanisms and total ionizing dose degradation. The concentrations and types of ions vary significantly with altitude and inclination of angle of an orbit, recent solar activity, and the amount of protective shielding. These variations make it difficult to specify a typical space environment. Most particles found in the space radiation environment of the earth can be classified as: (1) trapped particles in the magnetic field of the 
earth-primarily protons and electrons; and (2) cosmic rays-heavy ions or protons of solar or galactic origin.

Power metal-oxide semiconductor-field-effect transistors (MOSFETs) have dominated the power device market since the 1980s and are found in most power supplies, DC-DC converters, and low voltage controllers. They are frequently selected for use in space-borne electronic systems. However, power MOSFETs, in the space environment, are susceptible to parametric degradation and to at least two catastrophic failure mechanisms that can be initiated by the passage of a single heavy ion through sensitive regions of the device structure. These failures are referred to as single-event burnout (SEB) or single-event gate rupture (SEGR).

Currently, the silicon trench (or UMOS) power MOSFET device design is supplanting the older vertical double-diffused power MOSFET (VDMOS) device design for many applications including low voltage switches. The radiation susceptibilities of the VDMOS technology have been studied and reported on extensively. However, at this time, there is much less data and analysis of heavy-ion degradation and/or failure of UMOS devices. Three mechanisms for heavy-ion degradation and/or failure due to heavy ion exposure have been reported for UMOS power transistors: (1) microdose; (2) single-event burnout (SEB); and (3) single-event gate rupture (SEGR).

This paper will summarize the state of knowledge on the degradation and/or failure of silicon trench (or UMOS) power transistors due to heavy-ion exposure (or energetic proton environments) such as encountered in spacecraft. The evolution of power MOSFET technology will be recounted and the current physical understanding of the interaction between heavy-ion radiation and the UMOS device technology as it applies to the three mechanisms above will be described. Designers of spaceborne power electronics systems that incorporate UMOS power MOSFET technology need this information in order to evaluate the survivability of their designs.

\section{Evolution of Power MOSFET Technology}

Several power MOSFET device designs had been explored by the early 1980s. In that time period, the Vertical Double-Diffused MOS structure (VDMOS or simply DMOS) [1] as illustrated in Figure 1 emerged as the dominant MOS power device technology. The first generation of macrocell VDMOS power transistors was introduced into the commercial market by International Rectifier. Generally, power MOSFET design and process technology has followed the evolution of CMOS technology following the leading edge with a time delay of three-to-five years.

In the 1990s, power MOSFET device design based on trench technology became feasible and held several advantages when compared to VDMOS [2,3]. The trench or UMOS transistors delivered an improved switching resistance ( $\left.\mathrm{R}_{\mathrm{DSON}}\right)$ and reduced gate charge when designed for products with a drain voltage capability lower than $100 \mathrm{~V}$. The silicon trench power MOSFET is the leading low-voltage technology in the market today and is offered by a number of commercial, power MOSFET suppliers.

UMOS power transistors are fabricated with trenches etched into the silicon; gate oxides are then grown along the trench sidewalls; and then the trenches are filled with polysilicon, which is the gate material. Incorporating a trench structure significantly increases the channel width when compared to the VDMOS power transistor, and also eliminates the JFET region (commonly referred to as the neck region in the vertical power MOSFET). In some trench technologies, a thicker oxide is used at the 
trench bottom to dramatically reduce the capacitance between the gate and drain and to enhance device switching performance.

Figure 1. Schematic cross-section of an n-channel vertical power MOSFET. After ref. [1].

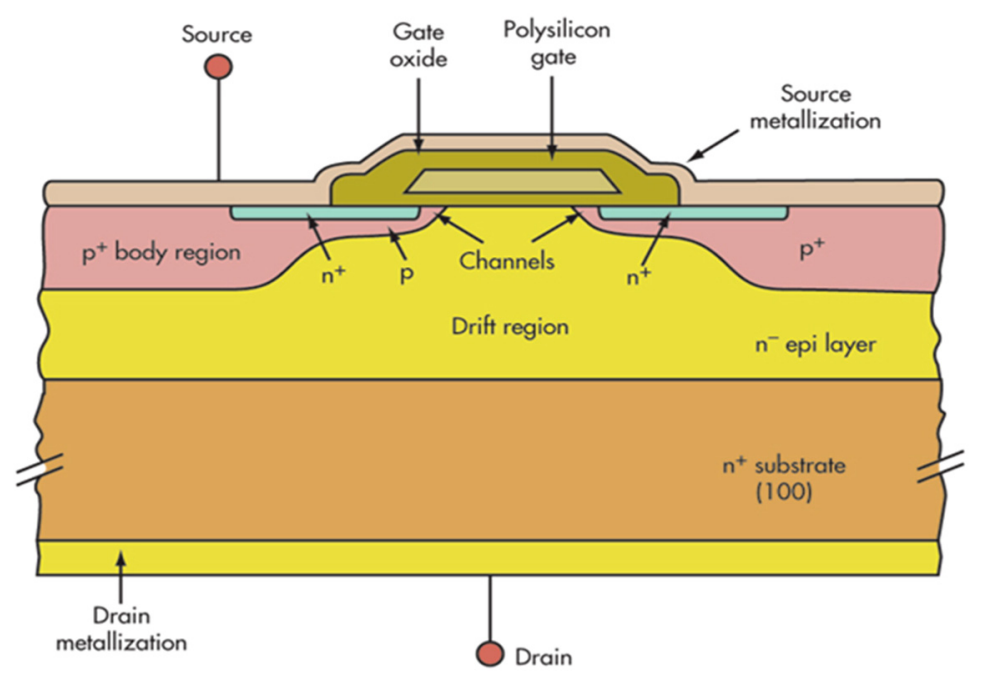

A cross-section of an n-channel UMOS power MOSFET showing typical device structure is given in Figure 2. Contact to the drain is made on the bottom surface of the silicon chip. The rather thick n-type epitaxial drain region (drift region) is required to drop the large drain to source voltages that the power transistor must block while operating in the OFF state. Typically, many cells (often laid out as stripes) are connected in parallel to effectively create a very wide channel (while retaining the channel length of the individual cell) to achieve the large currents required in the ON state. When turned ON, the channel forms and current flows from the source through the channel to the drain. Note the parasitic npn bipolar-junction-transistor (BJT) inherent to the structure. The source, body, and drain regions of the MOSFET comprise the emitter, base, and collector regions of the parasitic BJT. During normal operation, this parasitic BJT is always turned off due to the common source-body metallization that shorts out the base-emitter junction.

Figure 2. Schematic cross-section illustrating the structure of an n-channel UMOS MOSFET. After ref. [4].

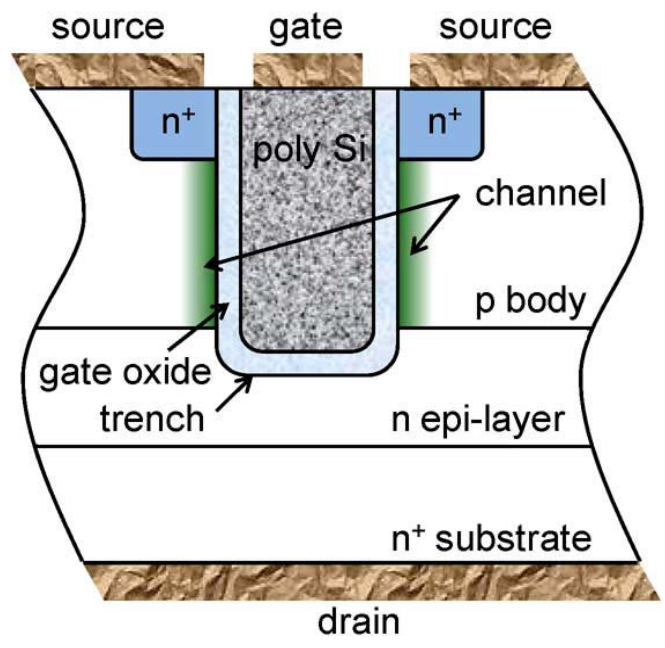




\section{Microdose Effects in UMOS Technology}

In 2008, S. Liu et al. [5] published a detailed analysis of total ionizing dose test results on commercial UMOS power MOSFETs illustrating that these devices have similar responses as radiation hardened VDMOS power devices but with, in general, larger parameter shifts. These tests were done with $\mathrm{Co}^{60}$ gamma rays. The changes are attributable to trapped charge in the gate oxide [6]. Liu et al. note that a radiation hardened gate oxide would mitigate most of the observed total dose effects observed.

However, ionizing radiation total dose effects from heavy ions and protons yield very different results when compared to $\mathrm{Co}^{60}$ tests. Felix et al. [7] reported enhanced degradation and microdose effects in UMOS power devices in 2007. Figure 3 illustrates measured shifts in the subthreshold I-V curves for a typical $30 \mathrm{~V}$ UMOS power MOSFET due to exposure to $\mathrm{Co}^{60}$ gamma rays. These particular devices are laid out in a stripe geometry with more than 1000 trenches yielding more than 2000 identical MOS transistors wired in parallel.

Figure 3. Typical subthreshold I-V curves for a $30 \mathrm{~V}$ UMOS device pre- and post $\mathrm{Co}^{60}$ radiation exposure. During exposure, $15 \mathrm{~V}$ was applied to the gate and all other pins grounded. After ref. [7].

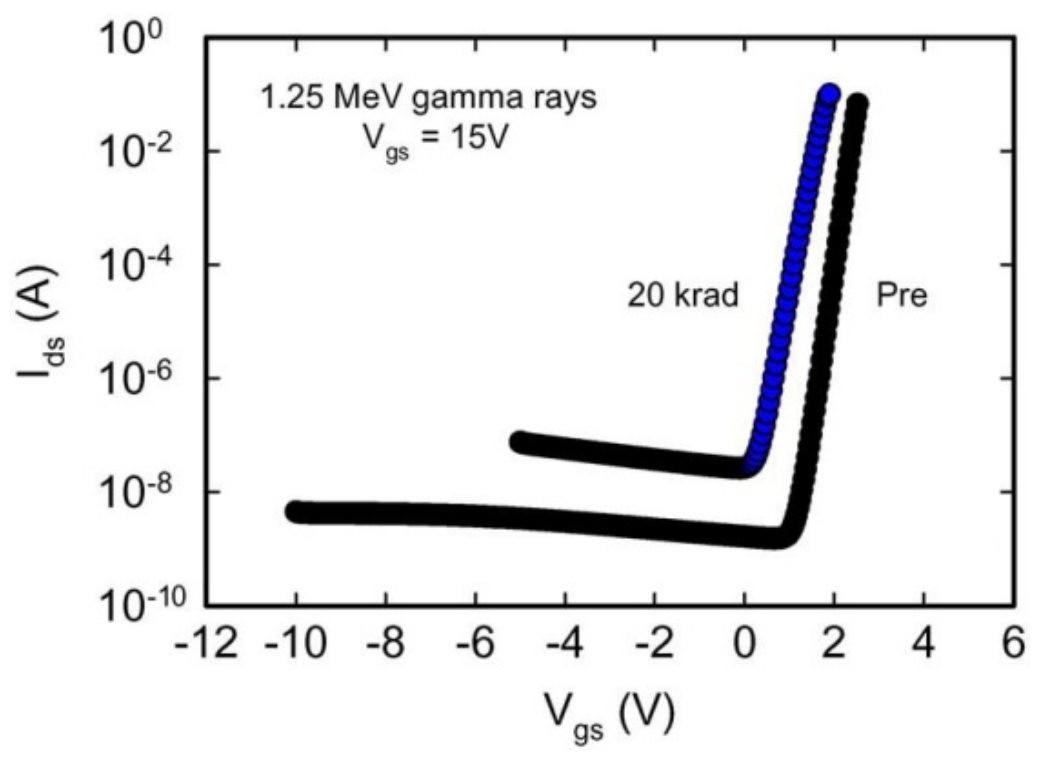

Figure 4 illustrates measured shifts in subthreshold I-V curves for samples of the same 30V UMOS power MOSFET type exposed to a beam of $2869 \mathrm{MeV}$ Xe ions. Felix et al. [7] present a detailed description of the experiment and a detailed analysis of the experimental results with the conclusion that the differences between the $\mathrm{Co}^{60}$ total dose $\mathrm{I}-\mathrm{V}$ results (Figure 3 ) and the heavy ion $\mathrm{I}-\mathrm{V}$ results (Figure 4) are due to parasitic transistors that result from microdose deposited by the heavy ions in the UMOS gate oxides. Small parasitic transistors are formed as a single heavy ion passes through the gate oxide generating electron-hole pairs with trapped positive charge resulting at the silicon-oxide interface [6]. 
Figure 4. Typical subthreshold I-V curves for a 30V UMOS device pre Xe heavy ion exposure and after exposure to several fluence levels. During exposure, 5V was applied to the gate and all other pins grounded. After ref. [7].

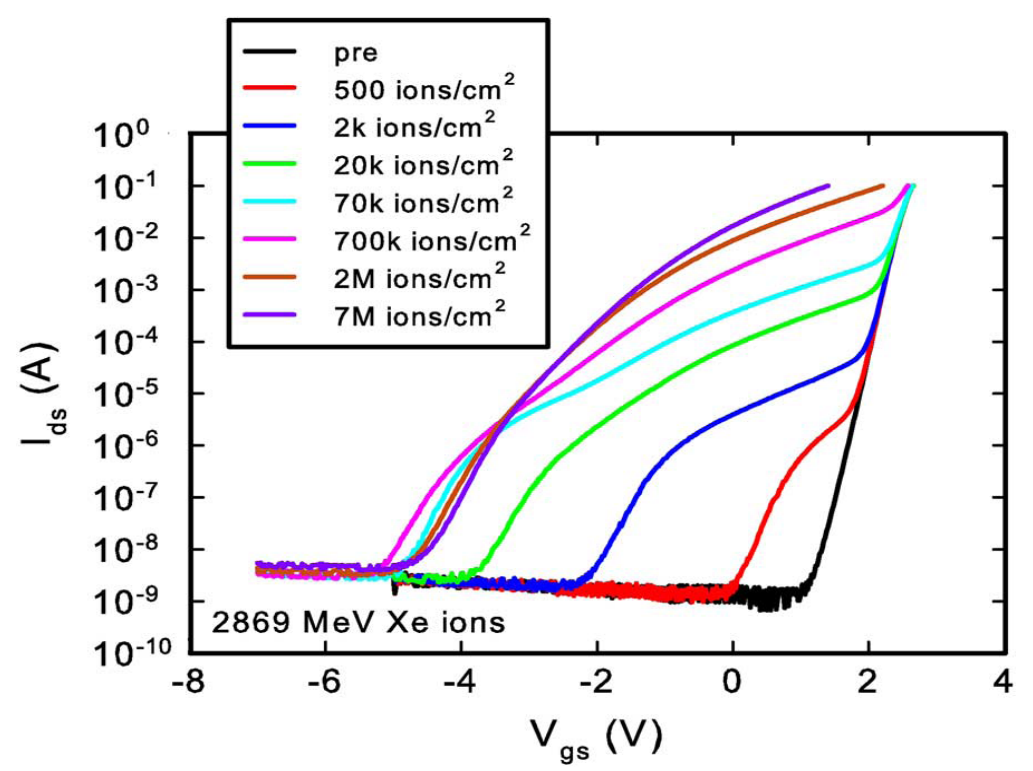

Formation of a parasitic transistor is illustrated in Figure 5, a cross section for a stripe geometry UMOS power device illustrating a heavy ion passing through the gate oxide along the trench sidewall. Trapped charge in the gate oxide, in the vicinity of the track, would reduce the threshold voltage resulting in unexpected off-state leakage currents.

Figure 5. Schematic cross section illustrating an ion strike through the gate oxide on the sidewall of the trench in a UMOS power device. After ref. [7].

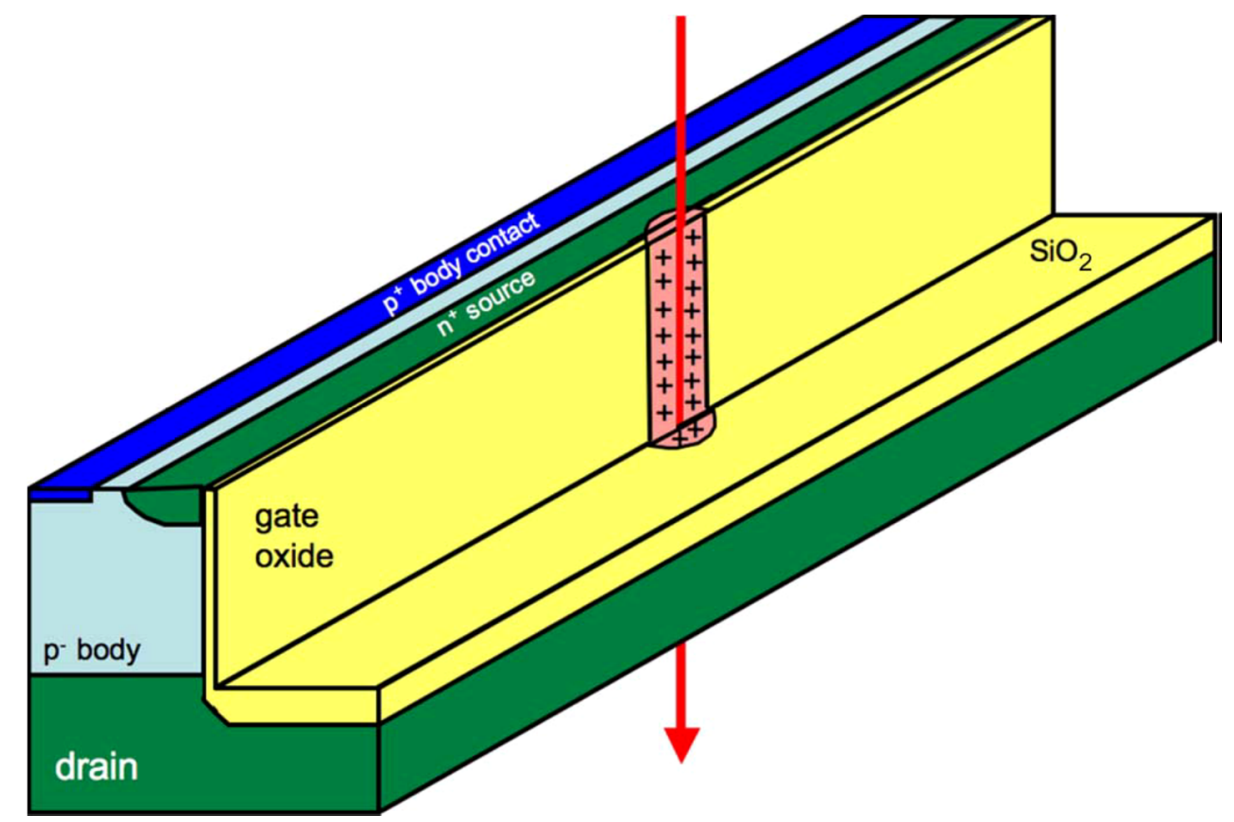

Other investigators have observed similar microdose effects in UMOS power devices $[4,8,9]$ and Griffoni et al. [10] have reported microdose effects induced by heavy ion strikes in the sidewall gate-oxide of FinFETs in advanced CMOS devices. 
In a 2008 paper, Shaneyfelt et al. [11] point out that “... if trench FET power MOSFETS are going to be used in proton-rich space environments, gamma ray testing of the devices could significantly underestimate the radiation-induced degradation, leading to unexpected failures in space environments ..."

The tests described here which illustrate the microdose effect were made with the ion beam incident perpendicular to the die surface. This convenient test geometry may not be representative of the direction of an ion (or proton) hit in an actual spaceborne system, thus possibly reducing the probability of this particular degradation mechanism.

\section{Single Event Burnout (SEB) Effects in UMOS Technology}

SEB in VDMOS and UMOS power devices is an effect dependent on the inherent parasitic BJT in the device structure. SEB is triggered when a heavy ion passes through the power MOSFET biased in the OFF state (blocking a high drain-source voltage). Short-lived transient currents generated by the heavy ion turn on the parasitic BJT [12,13]. Due to a regenerative feedback mechanism, collector currents in the BJT increase to the point where second breakdown sets in, creating a permanent short between the source and drain and rendering the MOSFET useless. A key component of the regenerative feedback mechanism is avalanche generated hole current in the collector region of the BJT. A good review of the SEB physics and the techniques used to model SEB in VDMOS power devices has been provided by Johnson et al. [14]. SEB in UMOS devices, because of the unique structure, is similar to SEB in BJTs [15].

SEB in UMOS power devices has been observed experimentally [9,16-18]. Liu et al. [17], in extensive tests, noted that the failure signatures of pure SEB failure observed in commercial trench power MOSFETs was very much like the SEB observed on radiation hardened VDMOSFETs. Koga et al. [9], in a 2014 paper, note similar results. A recent review paper by Titus [19] describes test circuits and methodology for such tests. During exposure to heavy ion radiation, the drain leakage current will suddenly increase to the allowed current limit while the gate leakage current remains at the base level. On visual inspection, burnout locations can often be found in the device active area.

Wang et al. [20] has used commercial TCAD software to simulate SEB in UMOS devices. Figure 6 is a cross section of a half-cell of the structure simulated with physical parameters indicated which approximate a UMOS device with breakdown voltage of $70 \mathrm{~V}$ and threshold voltage of $3 \mathrm{~V}$. The authors report that, as expected, drain-source voltage, ion strike position, and LET all figure in determining SEB thresholds and present tables illustrating the effects of varying these parameters on SEB. Figure 7 , from this work, shows the onset of SEB and the sensitivity to drain-source voltage for the ion strike through the gate poly.

For these simulations, the ion strike is assumed to be incident perpendicular to the die. The UMOS SEB simulations [20] do not explore the results of off perpendicular incident ions. Worst case response may not occur with the incident ion perpendicular to the die surface, but the worst case may be at beam angles almost parallel to the die surface [21]. 
Figure 6. Cross sectional view and doping concentrations used in the Wang et al. TCAD simulations reported in ref. [20].

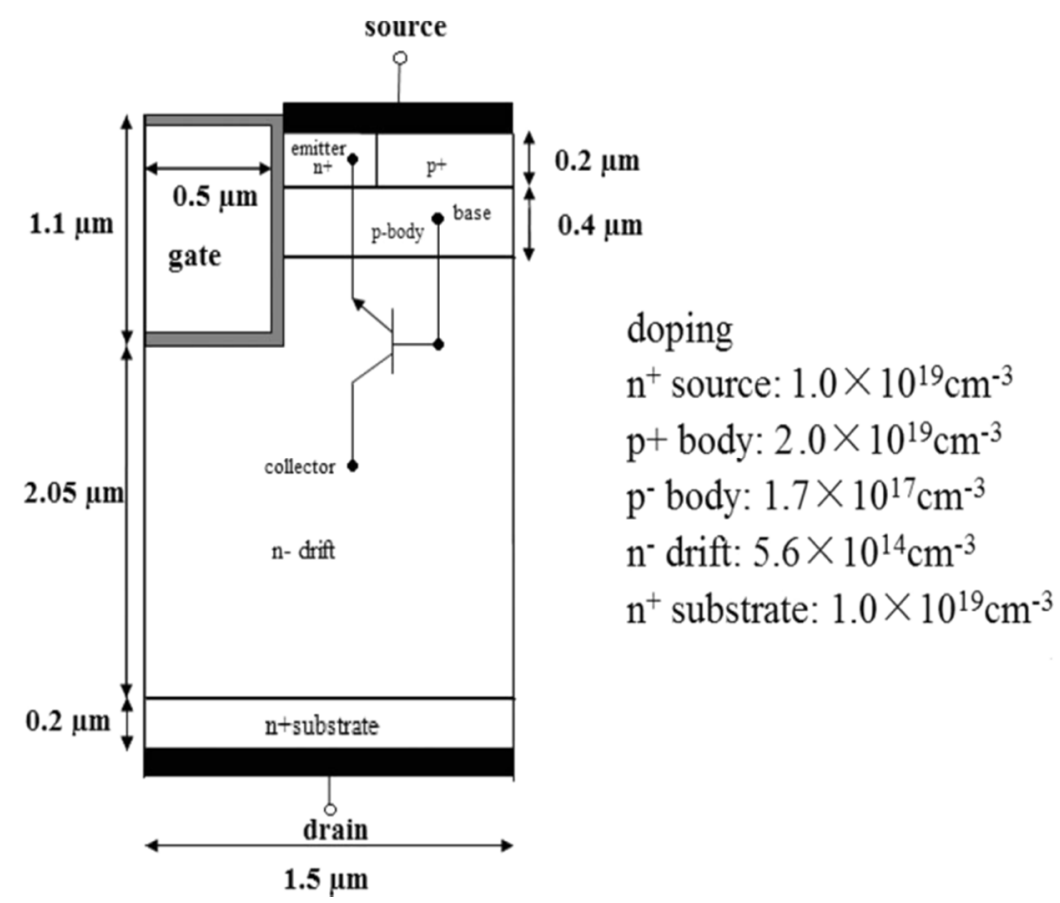

Figure 7. Drain current versus time for a 70V UMOSFET illustrating the on-set of SEB and the sensitivity to drain-source voltage. Ion strike is assumed to be incident perpendicular to the die and near the center of the trench. After ref. [20].

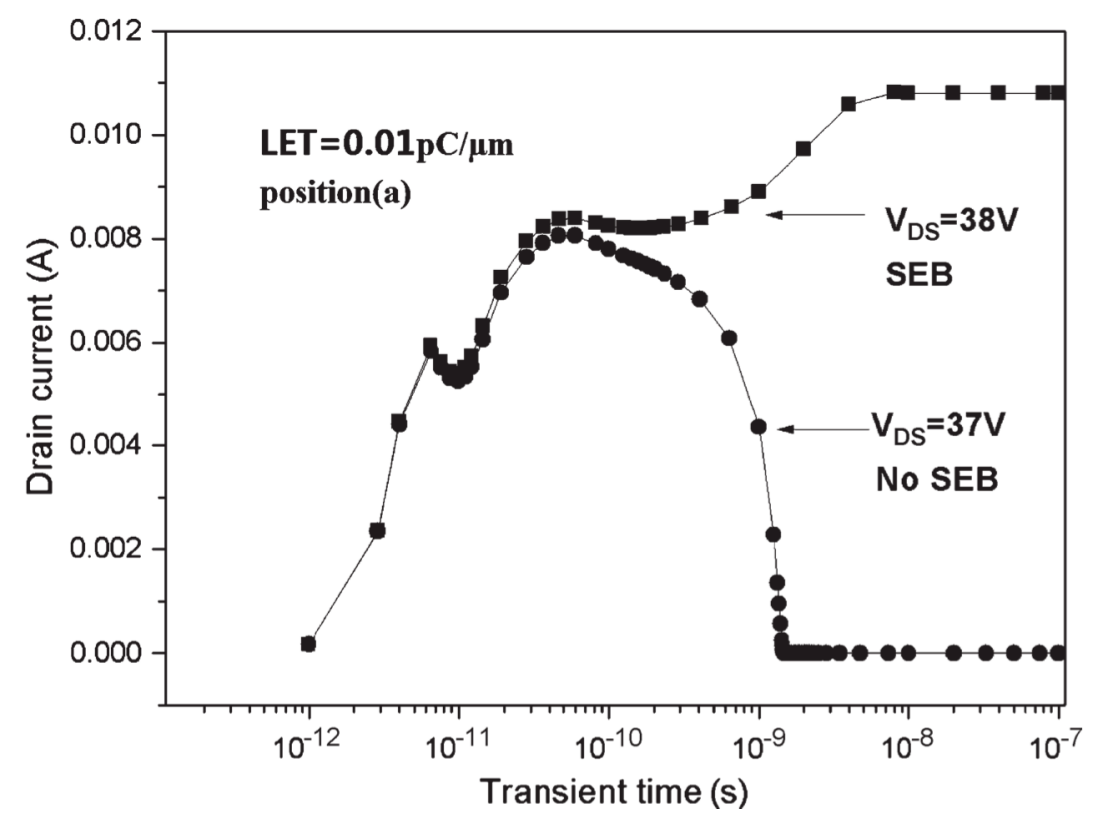

Wang et al. in other work [22] have used TCAD simulation to explore SEB hardening strategies for UMOSFETs. Simulations indicated that enlarging the $\mathrm{p}^{+}$plug and the addition of a buffer layer between the epitaxial layer and substrate can reduce SEB susceptibility with the buffer layer making the most difference. These results are similar to results obtained for VDMOS devices [19]. Using TCAD simulation, Wang et al. [23] have also been able to explore the potential for hardening using alternate structural configurations such as including a Schottky source plus an N-type buffer layer. 
They report that the threshold voltages for burnout are markedly increased making the devices less sensitive to SEB when compared to the burnout voltage of the base UMOS structures simulated [20].

\section{Single Event Gate Rupture (SEGR) in UMOS Technology}

SEGR is due to localized dielectric breakdown of the gate oxide [24-26]. Under appropriate bias conditions, accumulation of charge (generated by the heavy ion) in the silicon at the silicon-silicon oxide $\left(\mathrm{Si}_{-} \mathrm{SiO}_{2}\right)$ interface can result in a sufficiently high electric field across the gate oxide to cause gate rupture. SEGR is a destructive effect. As soon as the minimum voltage (SEGR threshold) required for SEGR to occur has been reached and passed, SEGR occurs almost instantly [26]. The peak electric field occurs in picoseconds of the ion passage through the structure. A good review of the SEGR physics and the techniques used to model SEGR in VDMOS power devices has been provided by Johnson et al. [14].

SEGR in UMOS power devices was experimentally observed by Koga et al. [9] and Liu et al. [16,17] in addition to measurements of SEB. Wang et al. [20] simulated SEGR in UMOS devices using the framework developed by Allenspach et al. [24,25]. Figure 6 is a cross section of a half-cell of the structure simulated.

In the Allenspach method, the critical electric field across the gate oxide required for onset of SEGR is related to the gate oxide thickness and the LET of the incident ion using a modified version of an empirical model first elucidated by Wrobel [27]. Then, 2-D simulations are used to determine (1) the peak electric field following the ion strike and (2) the pre-strike field in the gate-oxide for a specified set of bias conditions and oxide thickness. The maximum transient component of the oxide electric field can then be computed and compared to the critical electric field required for the onset of SEGR. This information allows mapping of bias conditions that allow safe operation or, conversely, device bias conditions that result in SEGR.

Figure 8 from [20] displays drain-source voltage that is the threshold for triggering SEGR or SEB in the 70V UMOSFET simulated as a function of incident ion LET. The ion strike is assumed to be incident perpendicular to the die and through the gate material near the center of the trench. Not surprisingly, the threshold for SEGR increases as LET decreases.

In a VDMOS device, the physical location of the actual gate rupture is assumed to be near where the ion strike passes through the gate oxide [28]. However, in the SEGR simulations of Wang et al. [20], the heavy ion is incident perpendicular to the die surface and passes through the gate poly and the bottom of the trench and the authors say that "a portion of the holes will be accumulated along the sidewalls of the trench near the bottom." This leaves some uncertainty as to the physical location of the gate rupture. Does the simulation indicate that the oxide breakdown occurs at the bottom of the trench or on the sidewall of the trench?

As in SEB failures, worst case response for SEGR may not occur with the incident ion perpendicular to the die surface, but the worst case may be at beam angles near parallel to the die surface [21]. Liu et al. [17] observed a higher probability of SEGR failure at beam angles tilted from the perpendicular. It would be interesting to see TCAD simulations of this failure extended to cases where the incident ion was not perpendicular to the UMOS device die. 
Figure 8. Threshold drain-source voltage for triggering SEGR or SEB in a $70 \mathrm{~V}$ UMOSFET as a function of incident ion LET. Ion strike is assumed to be incident perpendicular to the die and near the center of the trench. After ref. [20].

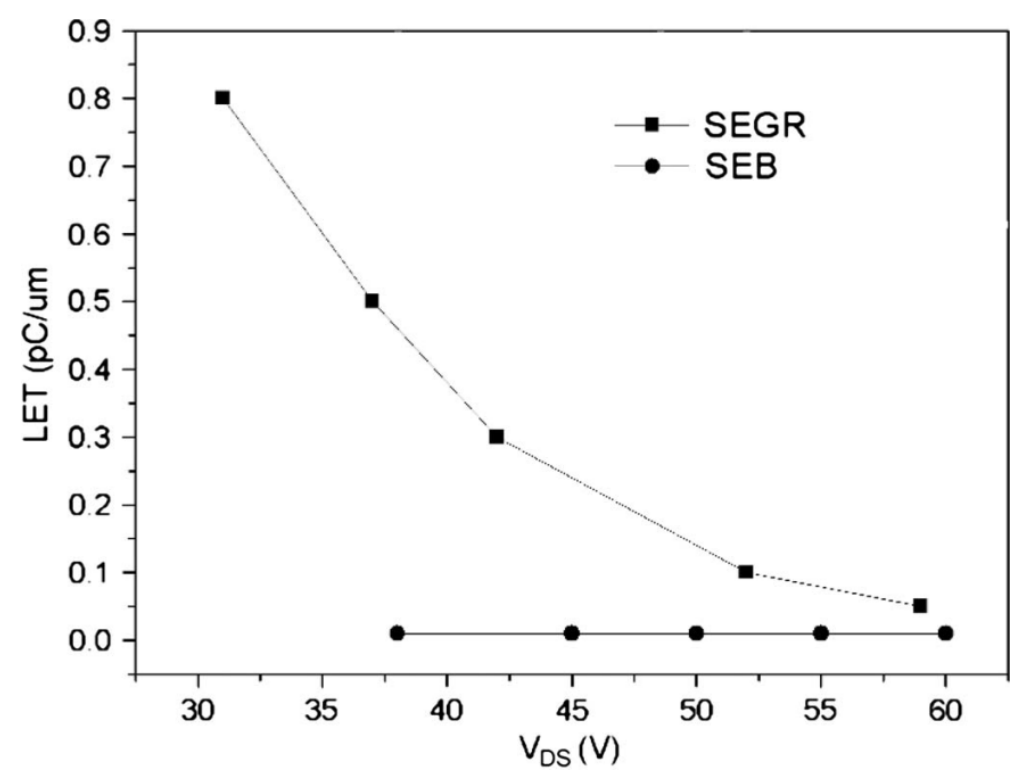

\section{Discussion}

UMOS power device technology has been shown by experiment to have vulnerabilities to SEB, SEGR, and microdose. Concern for the heavy-ion vulnerability of UMOS power devices in comparison to similarly rated VDMOS devices has been expressed [7,11,16]. Liu et al. [16] examined several devices in the 30-200 V range. The Lauenstein et al. [18] results were for a $250 \mathrm{~V}$ device. Neither group has published detailed experimental results in the open literature. Koga et al. [9] present results for two different $100 \mathrm{~V}$ devices with cross-section data and $\mathrm{I}-\mathrm{V}$ data. No research team has published detailed radiation-effects comparisons of UMOS devices with similarly rated VDMOS devices. The simulations by Wang et al. [20] are for a hypothetical $70 \mathrm{~V}$ device; there are no comparisons with experimental results.

Since there are significant differences in device structure and device physics between the VDMOS and UMOS technologies, understanding of the physical mechanisms of heavy-ion radiation degradation and failure of silicon UMOS power transistors would benefit from the publication of more detailed experimental studies of SEB and SEGR in UMOSFETs. This data coupled with TCAD modeling informed by experimental results should provide insights for improving UMOS device designs for survivability and provide validated protocols for utilization of these devices in spaceborne systems. Nevertheless, some radiation tolerant UMOS power products have been introduced in the commercial marketplace.

Additionally, with evidence that device bias voltages at which SEB and SEGR occurs in VDMOS (or the determination of a device's safe operating area) may be linked to the ion energy and ion species $[19,29,30]$ rather than linked solely to the LET of the ion, worst-case conditions for radiation hardness assurance testing and qualification of UMOS power transistors for use in space should be carefully examined. 


\section{Conclusion}

This review has summarizes the current state of knowledge regarding the degradation and/or failure of silicon trench (or UMOS) power transistors due to heavy-ion exposure (or energetic proton environments) such as encountered in spacecraft. Published work has demonstrated UMOS power device technology vulnerabilities to SEB, SEGR, and microdose. The physics of microdose degradation is well understood. The physics of SEB and SEGR failure of UMOS devices appears to be similar to that of VDMOS devices; however, some interesting questions remain. Additional experimental results and refinement of the models for simulation would allow improvement of UMOS device designs for survivability and provide guidelines for selection of these devices for spaceborne systems.

\section{Acknowledgments}

The author is grateful for technical discussions regarding UMOS power devices with R.D. Schrimpf and J.L. Titus.

\section{Conflicts of Interest}

The author declares no conflict of interest.

\section{References}

1. Grant, D.A.; Gowar, J. Power MOSFETs: Theory and Applications; John Wiley \& Sons: New York, NY, USA, 1989.

2. Shenai, K. Optimized Trench MOSFET Technologies for Power Devices. IEEE Trans. Electron Dev. 1992, 39, 1435-1443.

3. Saxena, R.S.; Kumar, M.J. Trench Gate Power MOSFET: Recent Advances and Innovations. In Advances in Microelectronics and Photonics; Jit, S., Ed.; Nova Science Publishers, Inc.: Hauppauge, NY, USA, 2012.

4. Kuboyama, S.; Maru, A.; Ikeda, N.; Hirao, T.; Tamura, T. Characterization of Microdose Damage Caused by Single Heavy Ion Observed in Trench Type Power MOSFETs. IEEE Trans. Nucl. Sci. 2010, 57, 3257-3261.

5. Liu, S.; DiCienzo, C.; Bliss, M.; Zafrani, M.; Boden, M.; Titus, J.L. Analysis of Commercial Trench Power MOSFETs' Responses to $\mathrm{Co}^{60}$ Irradiation. IEEE Trans. Nucl. Sci. 2008, 55, 3231-3236.

6. Swank, J.R.; Shaneyfelt, M.R.; Fleetwood, D.M.; Felix, J.A.; Dodd, P.E.; Paillet, P.; Ferlet-Cavrois, V. Radiation Effects in MOS Oxides. IEEE Trans. Nucl. Sci. 2008, 55, 1833-1853.

7. Felix, J.A.; Shaneyfelt, M.R.; Schwank, J.R.; Dalton, S.M.; Dodd, P.E.; Witcher, J.B. Enhanced Degradation in Power MOSFET Devices Due to Heavy Ion Irradiation. IEEE Trans. Nucl. Sci. 2007, 54, 2181-2189.

8. Yan, Y. Dept. of Engineering Physics, Tsinghua University, Private communication.

9. Koga, K.; Bielat, S.; George, J. Charged Particle Induced Degradation of Trench Type n-channel Power MOSFETs. IEEE Radiation Effects Data Workshop Record, Paris, France, 16 July 2014, (to be published). 
10. Griffoni, A.; Gerardin, S.; Roussel, P.J.; Degraeve, R.; Meneghesso, G.; Paccagnella, A.; Simoen, E.; Claeys, C. A Statistical Approach to Microdose Induced Degradation in FinFET Devices. IEEE Trans. Nucl. Sci. 2009, 56, 3285-3292.

11. Shaneyfelt, M.R.; Felix, J.A.; Dodd, P.E.; Schwank, J.R.; Dalton, S.M.; Baggio, J.; Ferlet-Cavrois, V.; Paillet, P.; Blackmore, E.W. Enhanced Proton and Neutron Induced Degradation and Its Impact on Hardness Assurance Testing. IEEE Trans. Nucl. Sci. 2008, 55, 3096-3104.

12. Hohl, J.H.; Johnson, G.H. Features of the Triggering Mechanism for Single Event Burnout of Power MOSFETs. IEEE Trans. Nucl. Sci. 1989, 36, 2260-2266.

13. Johnson, G.H.; Hohl, J.H.; Schrimpf, R.D.; Galloway, K.F. Simulating Single-Event Burnout on N-Channel Power MOSFETs. IEEE Trans. Electron. Dev. 1993, 40, 1001-1008.

14. Johnson, G.H.; Palau, J.M.; Dachs, C.; Galloway, K.F.; Schrimpf, R.D. A Review of the Techniques Used for Modeling Single-Event Effects in Power MOSFET's. IEEE Trans. Nucl. Sci. 1996, 43, 546-560.

15. Titus, J.L.; Johnson, G.H.; Schrimpf, R.D.; Galloway, K.F. Single-Event Burnout of Power Bipolar Junction Transistors. IEEE Trans. Nucl. Sci. 1991, 38, 1315-1322.

16. Liu, S.; Zafrani, M.; Cao, H.; Berberian, R.; DiCienzo, C.; Boden, M. Vulnerable Trench Power MOSFETs under Heavy Ion Irradiation. Presented at the 2008 IEEE Nuclear and Space Radiation Effects Conference, Tucson, AZ, USA, 14-18 July 2008, (unpublished).

17. Liu, S.; Cao, H.; Berberian, R.; DiCienzo, C.; Zafrani, M. SEE Test Results \& Observed Failure Modes of Trench Power MOSFETs. Presented at the 2008 IEEE Nuclear and Space Radiation Effects Conference, Tucson, AZ, USA, 14-18 July 2008, (unpublished).

18. Lauenstein, J.-M.; Topper, A.D.; Casey, M.C.; Wilcox, E.P.; Phan, A.M.; Kim, H.S.; LaBel, K.A. Recent Radiation Test Results for Power MOSFETs. IEEE Radiation Effects Data Workshop Record, San Francisco, CA, USA, 11 July 2013, pp. 183-188.

19. Titus, J.L. An Updated Perspective of Single Event Gate Rupture and Single Event Burnout in Power MOSFETs. IEEE Trans. Nucl. Sci. 2013, 60, 1912-1928.

20. Wang, Y.; Zhang, Y.; Yu, C. Research of Single-Event Burnout in Power UMOSFETs. IEEE Trans. Electron. Dev. 2013, 60, 887-892.

21. Titus, J.L. NSWC Crane, Private communication.

22. Wang, Y.; Zhang, Y.; Wang, L.-G.; Yu, C. Single-Event Burnout Hardening of Power UMOSFETs with Optimized Structure. IEEE Trans. Electron. Dev. 2013, 60, 2001-2007.

23. Wang, Y.; Zhang, Y.; Cao, F.; Shan, M.-G. Single-Event Burnout Hardened Structure of Power UMOSFETs with Schottky Source. IEEE Trans. Power Electron. 2014, 29, 3733-3737.

24. Allenspach, M.; Brews, J.R.; Mouret, I.; Schrimpf, R.D.; Galloway, K.F. Evaluation of SEGR threshold in power MOSFETs. IEEE Trans. Nucl. Sci. 1994, 41, 2160-2166.

25. Allenspach, M.; Mouret, I.; Titus, J.L.; Wheatley, C.F.; Pease, R.L.; Brews, J.R.; Schrimpf, R.D.; Galloway, K.F. Single-event gate rupture in power MOSFETs: Prediction of breakdown biases and evaluation of oxide thickness dependence. IEEE Trans. Nucl. Sci. 1995, 42, 1922-1927.

26. Mouret, I.; Calvel, P.; Allenspach, M.; Titus, J.L.; Wheatley, C.F.; LaBel, K.A.; Calvet, M.-C.; Schrimpf, R.D.; Galloway, K.F. Measurement of a Cross-Section for Single-Event Gate Rupture in Power MOSFET's. IEEE Electron. Dev. Lett. 1996, 17, 163-165. 
27. Wrobel, T.F. On heavy ion induced hard-errors in dielectric structures. IEEE Trans. Nucl. Sci. 1987, 34, 1262-1268.

28. Brews, J.R.; Allenspach, M.; Schrimpf, R.D.; Galloway, K.F.; Titus, J.L.; Wheatley, C.F. A Conceptual Model of Single-Event Gate-Rupture in Power MOSFET's. IEEE Trans. Nucl. Sci. 1993, 40, 1959-1966.

29. Liu, S.; Lauenstein, J.-M.; Ferlet-Cavrois, V.; Marec, R.; Hernandez, F.L.; Scheick, L.; Bezerra, F.; Muschitiello, M.; Poivey, C.; Sukhaseum, N.; et al. Effects of Ion Species on SEB Failure Voltage of Power MOSFETs. IEEE Trans. Nucl. Sci. 2011, 58, 2991-2997.

30. Ferlet-Cavrois, V.; Schwank, J.R.; Liu, S.; Muschitiello, M.; Beutier, Th.; Javanainen, A.; Hedlund, A.; Poivey, C.; Zadeh, A.; Harboe-Sorensen, R.; et al. Influence of Beam Conditions and Energy for SEE Testing. IEEE Trans. Nucl. Sci. 2012, 59, 1149-1160.

(C) 2014 by the authors; licensee MDPI, Basel, Switzerland. This article is an open access article distributed under the terms and conditions of the Creative Commons Attribution license (http://creativecommons.org/licenses/by/4.0/). 